\title{
Peptide and protein analysis with mass spectrometry
}

\author{
Sunia A. Trauger, William Webb and Gary Siuzdak* \\ The Scripps Research Institute Center for Mass Spectrometry and Department of Molecular Biology, \\ The Scripps Research Institute, 10550 North Torrey Pines Road, La Jolla, CA 92037, USA
}

\begin{abstract}
Mass spectrometry (MS) is rapidly becoming a fundamental tool for biologists and biochemists in their efforts to characterize cellular function. Recent advancements in MS technology and front-end methodologies, along with the completion of the human genome have greatly popularized its use by researchers for protein identification and characterization. This paper is a general overview of how mass spectrometry is being used for the analysis of peptides and proteins, focusing on its application to molecular weight determination. Sample preparatory and cleanup techniques used in our laboratory for protein and peptide analysis are provided, along with a discussion of data interpretation.

The utility of mass spectrometry for protein and peptide analyses lies in its ability to provide highly accurate molecular weight information on intact molecules. The ability to generate such accurate information can be extremely useful for protein identification and characterization. For example, a protein can often be unambiguously identified by the accurate mass analysis of its constituent peptides produced by either chemical or enzymatic treatment of the sample. Furthermore, protein identification can also be facilitated by analysis of the protein's proteolytic peptide fragments in the gas phase; fragment ions generated inside the mass spectrometer via collision-induced dissociation (CID) to yield information about the primary structure and modifications. This overview describes how electrospray ionization (ESI) and matrix-assisted laser desorption/ionization (MALDI) mass spectrometry is being used for peptide and protein characterization focusing on its application to molecular weight determination.
\end{abstract}

\section{General overview of ESI and MALDI-MS for peptides and proteins}

What has made the mass analysis of peptides and proteins possible is the ability to promote their nondestructive vaporization/ionization through either ESI [1-3], MALDI [4,5] or Desorption/Ionization on Silicon (DIOS) [6]. Ionization for these methods occurs through the addition or removal of protons such as [peptide $+\mathrm{H}]^{+}$, [peptide $\left.+2 \mathrm{H}\right]^{2+}$, or [peptide $\left.+n \mathrm{H}\right]^{n+}$ or [peptide $\left.-\mathrm{H}\right]^{-}$(Fig. 1). In the generation of these species it is fortunate that little to no fragmentation is observed by either ESI, MALDI, and DIOS for peptides and virtually no fragmentation is observed for proteins. As a result the generation of molecular weight information on peptides and proteins is quite unambiguous.

In general the sensitivity of mass spectrometry is excellent for peptides and proteins. It is possible to observe peptides at the attomole level [3]. The sensitivity for protein analysis is typically lower because of the protein's larger size. Proteins strike the detector with lower velocity than smaller molecules resulting in less secondary electron emission. Even so, the sensitivity of MALDI and ESI for proteins is still considered good at the picomole and femtomole level.

In addition to molecular weight determination (Fig. 2), where MALDI, ESI, and DIOS have demonstrated increasing utility is in the identification of proteins [1-8] and protein post-translational modifications [9]. While complete and routine sequence determination through mass analysis of proteins is yet to be realized, it is now possible to use proteolytic peptide fragments in combination with database

${ }^{*}$ Corresponding author. E-mail: siuzdak@ scripps.edu. 

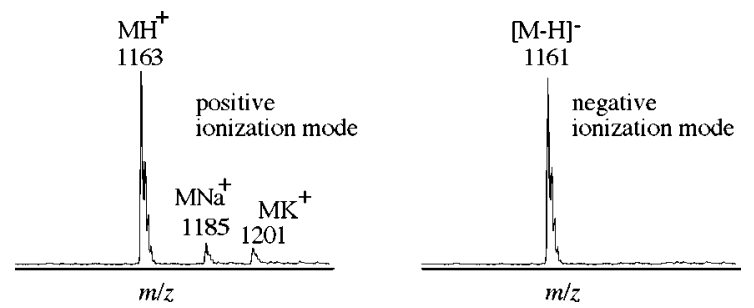

Fig. 1. Electrospray ionization mass spectra of a peptide in positive and negative ionization modes.

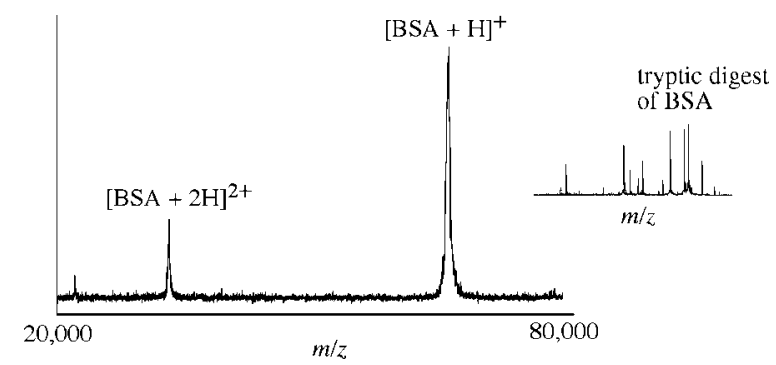

Fig. 2. A typical MALDI mass spectrum of the protein bovine serum albumin (BSA) with inset of the tryptic digest of BSA.

searching algorithms to identify proteins. This is accomplished by combining mass spectrometry with enzymatic or chemical digestion (Fig. 2 insert) followed by mass analysis of the peptide products and database searching techniques (especially useful in the post genome age).

Mass spectrometry has also demonstrated its utility in identifying post-translational modifications, as these modifications usually lead to a predictable change in molecular weight. In addition, electrospray with tandem mass spectrometry is demonstrating a capacity for performing de novo sequencing. This manuscript focuses on the current analysis capabilities in peptide and protein mass spectrometry, some specific preparation procedures, matrix selection, and the application of MALDI and ESI for protein identification and post-translational modifications.

\section{Peptides and proteins by MALDI}

MALDI is useful for peptide and protein analysis for the following reasons:

(1) MALDI allows for rapid sample analysis (using multi-sample plates, Fig. 3);

(2) high sensitivity (in the femtomole range or lower);

(3) tolerant of heterogeneous samples such as proteolytic digests (Figs 2 and 4);

(4) highly accurate mass measurements can now be generated in the low mass range below $3000 \mathrm{~m} / \mathrm{z}$;

(5) excellent dynamic range (Fig. 5).

MALDI mass analysis allows for rapid analysis primarily because it allows for the preparation of multiple samples simultaneously through the use of multi-sample probes. The automation of MALDI analyses is becoming increasingly important in proteomics and combinatorial chemistry. These analyses are driven by a computer-controlled procedure to monitor the ion signal as a function of laser position and laser intensity (Fig. 3). To accomplish this, the computer workstation automatically adjusts laser intensity and searches the sample well until a signal (within the specified mass range and intensity threshold) is 


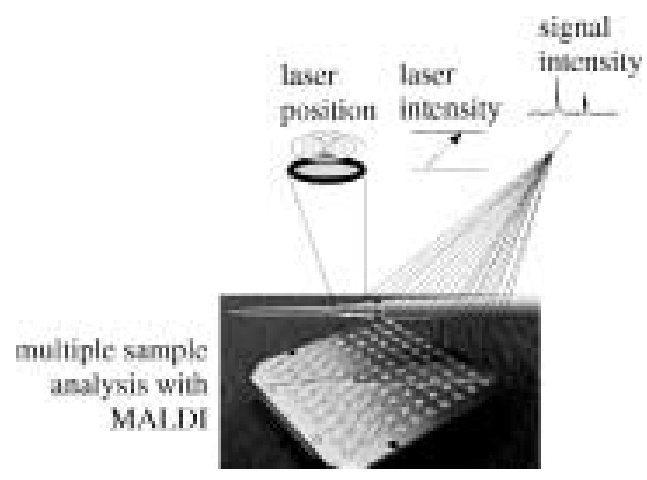

Fig. 3. The MALDI sample plate can be used for multisample preparation and automated sample analysis. Three different parameters can be used to adjust and monitor MALDI autosampling including: laser position, laser intensity, signal intensity, and mass range.

obtained. Based on a careful preselection of autosampler options, each parameter (laser intensity, search pattern, step size in well, signal intensity, and $\mathrm{m} / z$ range) is adjusted to minimize time of analysis and maximize signal quality.

For MALDI analysis of relatively small molecules and peptides (200 Da $<$ samples $<1000 \mathrm{Da}$ ), the 2,5-dihydroxybenzoic acid (DHB) matrix produces only a minimal amount of interference in the low molecular weight range and it is particularly well suited for generating low mass ions ( $<3000 \mathrm{Da})$. Other matrices such as are $\alpha$-cyano-4-hydroxycinnamic acid ( $\alpha$-cyano) give a high matrix background although they are particularly good for generating ions above 700 Daltons. For peptides generated by enzymatic digestion, $\alpha$-cyano is often the matrix of choice. 3,5-dimethoxy-4-hydroxycinnamic acid (sinapinic acid), another common matrix, is most commonly used for whole protein analysis. Most matrices reported to date have been acidic, but basic matrices have also been introduced, such as the 2-amino-4-methyl-5nitropyridine matrix, which extends the utility of MALDI to acid sensitive peptides, proteins, or other acid-sensitive compounds. Interesting different matrices can provide dramatically different results on the same sample (Fig. 4).

\section{Typical MALDI sample preparation procedures}

\subsection{Whole proteins}

- A saturated solution of $\alpha$-cyano or sinapinic acid can be prepared in acetonitrile, water and TFA (50/50 acetonitrile/water with $0.1 \%$ TFA). A saturated solution of $\alpha$-cyano and sinapinic acid can also be used where they are dissolved in acetonitrile, water and TFA (acetonitrile/water/TFA $=1: 1: 0.1$ ) and are vortexed for a complete mixing. The solution is ready for use when the undissolved solid settles. Matrices may be washed with $300 \mathrm{ul}$ water/0.2\% TFA solution prior to final preparation for additional salt removal. $30 \mathrm{mM}$ DHB in $\mathrm{MeOH}$ or acetonitrile-water $(1: 1)$ can also be used as the DHB matrix solution.

- Place $0.5 \mathrm{ml}$ protein solution (1-10 pmol/ml) directly on plate and then add $0.5 \mathrm{ml}$ matrix solution.

\subsection{Protein digests}

- Digest conditions include mixing $2.5 \mu \mathrm{l}$ protein $(1 \mu \mathrm{g} / \mu \mathrm{l}), 0.4 \mu \mathrm{l}$ trypsin $(0.1 \mu \mathrm{g} / \mu \mathrm{l}), 1.25 \mu \mathrm{l}$ $\mathrm{NH}_{4} \mathrm{HCO}_{3}(200 \mathrm{mM})$, with $6 \mu \mathrm{l} \mathrm{H}{ }_{2} \mathrm{O}$ and digest at $37^{\circ} \mathrm{C}$ overnight. 


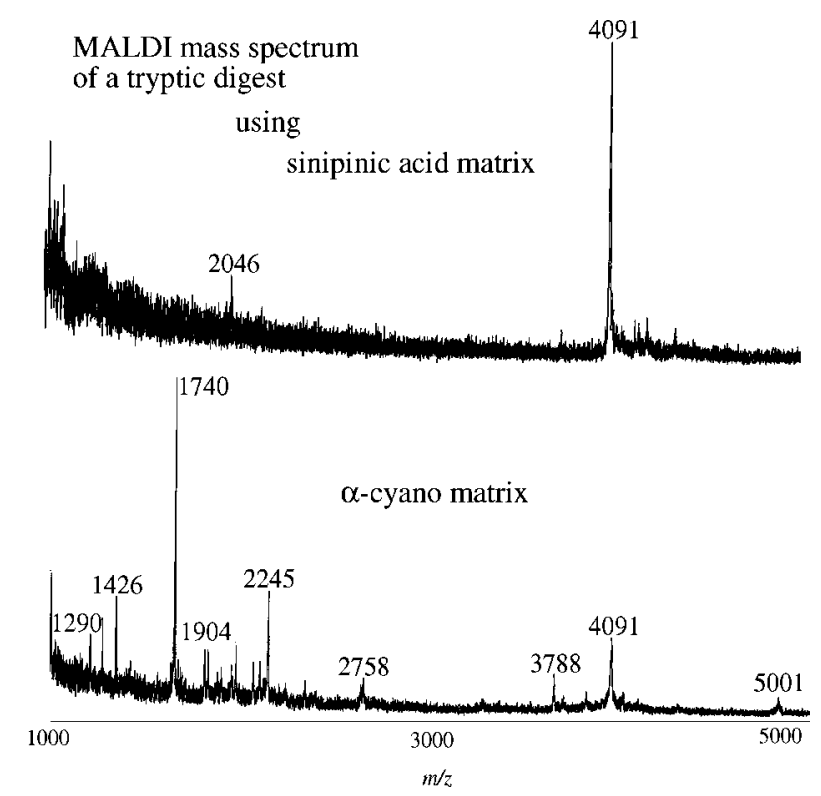

Fig. 4. MALDI-MS analysis of the same tryptic digest of a protein: different matrices can provide significantly different MALDI results on the same sample.

- In preparation for MALDI analysis mix $0.5 \mu$ l of proteolytic peptide solution $(\sim 1 \mathrm{pmol} / \mu \mathrm{l})$ on plate and then immediately add $0.5 \mu \mathrm{l}$ matrix solution. Allow to dry.

Target cleaning is one of the basic steps in sample preparation; we typically use methanol saturated cotton swabs and Kimwipes ${ }^{\mathrm{TM}}$ to remove sample residues on the target surface. The target is then sonicated for $\sim 15$ minutes in $\mathrm{MeOH} / \mathrm{H}_{2} \mathrm{O} /$ acetic acid $(1: 1: 0.1 \% \mathrm{v} / \mathrm{v})$ solution followed by another 15 minutes sonication in $\mathrm{MeOH} / \mathrm{H}_{2} \mathrm{O}$. This procedure should be repeated till no visible residues are left on the target.

In the dry drop method of sample crystallization where 0.5 to 1.0 picomole is introduced onto a MALDI plate: An equal amount of matrix solution is then added to the sample and the mixture is set to dry at room temperature. A gentle stream of cold air from a blower, or placing sample in a 37 degree incubator, can assist sample drying. Washing the dried sample/matrix with cold water can serve to remove contaminants like salts and detergents. An example of washing the sample after it has been deposited on the matrix is shown in Fig. 5.

\section{On-plate sample wash}

As can be seen from Fig. 5, on plate washes with cold water can be quite effective for removing salts from MALDI matrix solutions. What follows is a simple washing procedure.

(1) Deposit $0.5 \mu \mathrm{l}$ of the analyte and then $0.5 \mu \mathrm{l}$ matrix and allow to dry.

(2) Load the sample and try it. Check crystal appearance in magnified view.

(3) If results and/or crystals are poor pipet 2-3 $\mu$ l of Milli-Q water on top of the sample.

(4) Allow the droplet to stand for $\sim 2$ minutes.

(5) Pipet the water droplet in and out a few times to wash the matrix solution surface. 

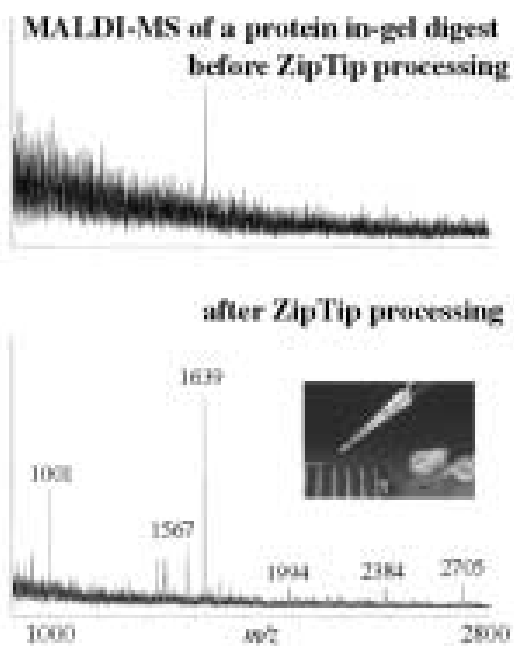

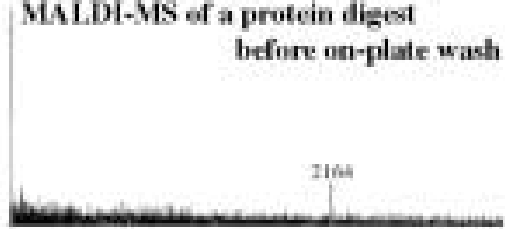

after an-plate wash

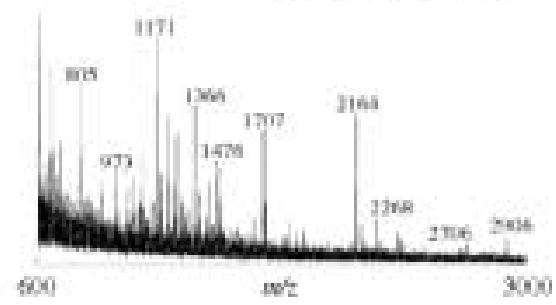

Fig. 5. (Left) MALDI-MS of a tryptic digest of 5 pmol of BSA before and after processing with C18 ZipTip. (Right) MALDI-MS before and after an on-plate wash of an unknown protein providing results that allowed it to be identified as mSin3A mouse protein using database searching.

(6) Pipet the water off the sample trying to remove as much as possible.

(7) It may be necessary to add a small amount of matrix.

(8) Allow to dry and analyze.

(9) The wash steps can be repeated if necessary, but do not add matrix more than once.

\section{ZipTip $^{\mathrm{TM}}$ sample wash}

Another popular approach for cleaning samples prior to analyzing them by MALDI or electrospray is using the ZipTip ${ }^{\mathrm{TM}}$. ZipTip ${ }^{\mathrm{TM}}$ pipette tips are a $10 \mu \mathrm{l}(\mathrm{P}-10)$ pipette tip with a bed of resin fixed at the end such that there is no dead volume. ZipTip ${ }^{\mathrm{TM}}$ Pipette Tips are useful for concentrating, desalting and fractionating picomole amounts of peptide, protein or oligonucleotide samples prior to analysis. In operation, ZipTips are affixed to a single or multi-channel pipette. Sample is aspirated and dispensed through the tip to bind, wash and elute the analyte(s) of interest. The concentrated, purified sample is precisely eluted in $1-4 \mu \mathrm{l}$ of eluant. The working volume range of $\mathrm{ZipTip}^{\mathrm{TM}}$ is between 1 to $100 \mu \mathrm{l}$ and availability includes $\mathrm{C} 18$ and $\mathrm{C} 4$ resins. The maximum molecular weight of a polypeptide which can be processed in ZipTip ${ }^{\mathrm{TM}} \mathrm{C} 18$ is typically $50,000 \mathrm{Da}$ and they can only be used to concentrate and desalt a sample once, which is performed in about a minute. For electrospray mass spectrometry the appropriate eluant for peptides is $50 \%$ ACN/ $0.1 \%$ TFA or $0.1 \%$ formic acid in $50 \%$ water, for proteins $75 \% \mathrm{MeOH} / 0.1 \%$ TFA or $0.1 \%$ formic acid in Milli-Q water. Elution volumes for ZipTip ${ }^{\mathrm{TM}}$ Pipette Tips are typically 2-4 $\mu$ l. A minimum sample amount for ZipTip ${ }^{\mathrm{TM}} \mathrm{C} 18$ is approximately 30 femtomoles of ACTH and angiotensin. In general, 1 pmol of sample should provide an adequate signal. Recoveries of $75 \%$ are typical for concentrations above $5 \mathrm{pmol} / \mu \mathrm{l}$, at concentrations of 2.5 to $0.25 \mathrm{pmol} / \mu \mathrm{l}$, recoveries of $45-50 \%$ have been observed.

In addition to excellent sensitivity MALDI has other useful features and capabilities. One of the most attractive features is its ability to analyze complex mixtures typically with less signal suppression than ESI (Figs 2 and 6), making it extremely useful for biological samples such as protein digests. An illus- 

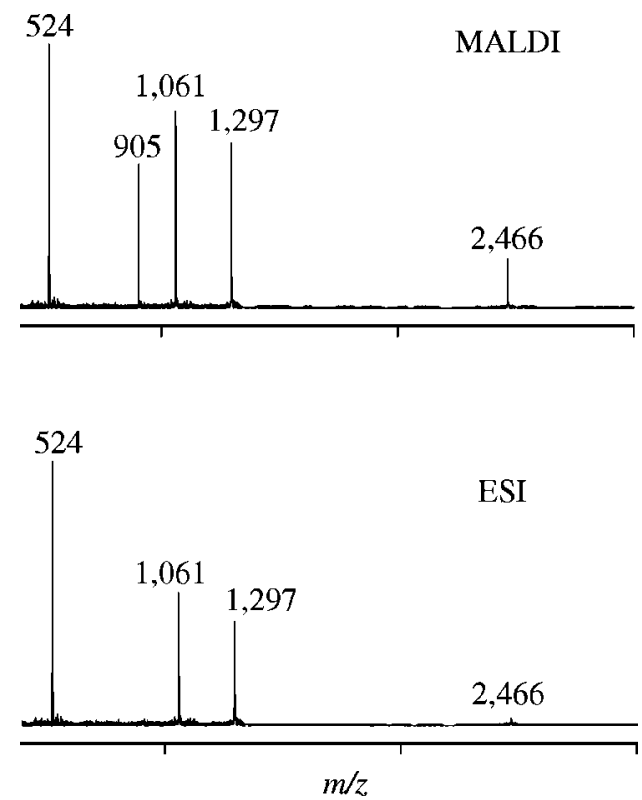

Fig. 6. MALDI mass spectrum of a five peptides illustrates its utility for mixture analysis, ESI mass spectral data of the same mixture gave significant signal suppression of the ions at $\mathrm{m} / \mathrm{z} 905$ and 2466 while MALDI provided a signal for all five peptides. This effect can vary significantly depending on sample, solution and analysis conditions. In general, MALDI provides better signals in simultaneous mixture analysis.

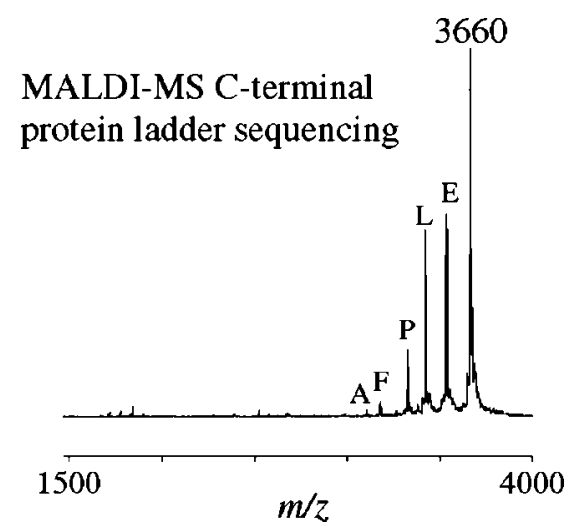

Fig. 7. The positive ion MALDI-MS ladder sequencing data using the carboxypeptidase Y digestion of the peptide ACTH (7-38) provided some $\mathrm{C}$-terminal sequence information.

tration (Fig. 7) of mixture analysis is shown with protein ladder sequencing, where a mixture of peptides/proteins differing in size by a single amino acid. This is primarily used for C-terminal sequencing with exopeptidase digestion.

Accuracy is also important. MALDI mass analysis (Figs 2-5) is typically performed with time-offlight (TOF) or TOF-reflectron analyzers with resolving capabilities on the order of 400 to 10,000 and accuracy ranging from $\pm 0.2 \%$ to $0.005 \%$. The resolution and accuracy depend upon the type of instrument being used (e.g., low resolution TOF versus a high resolution TOF reflectron or FTMS [10]), the presence of an internal standard, the size/type of analyte, and the selection of matrix material. 


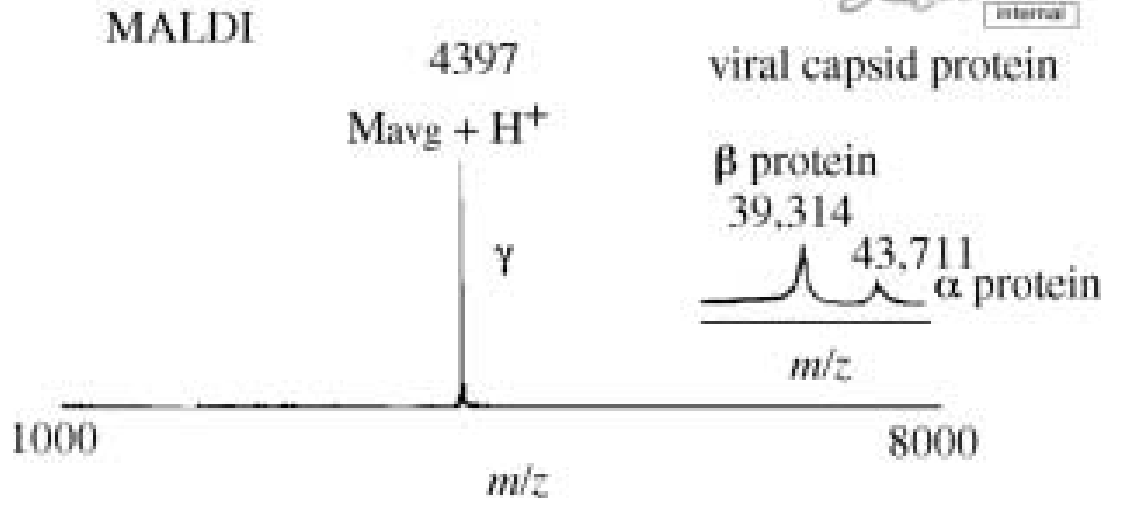

Fig. 8. MALDI time-of-flight MS data showing viral capsid proteins using sinapinic acid matrix. The data demonstrates the simultaneous acquisition of proteins of significantly differing size.

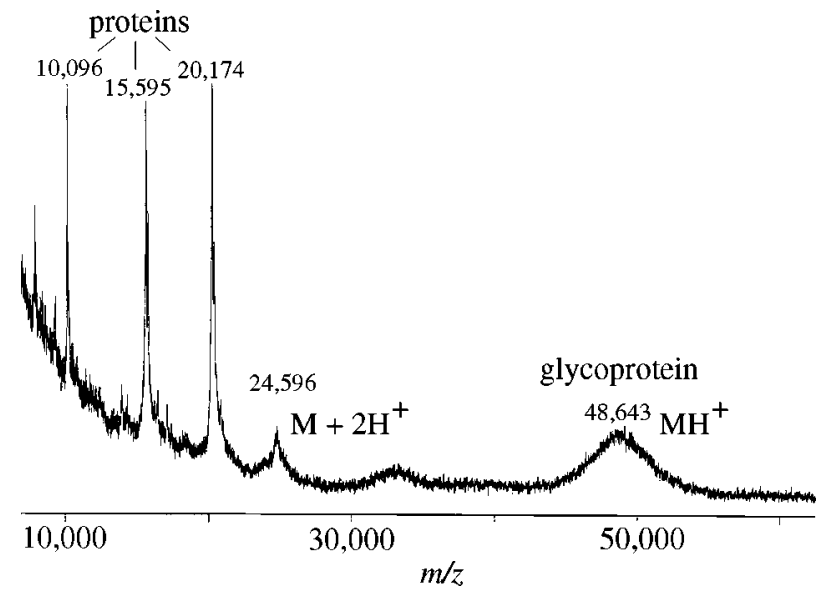

Fig. 9. MALDI time-of-flight MS data showing a glycoprotein and proteins containing no glycosylation. Note the significant difference in the broadness of the glycoprotein peak due to carbohydrate heterogeneity.

Another important feature is mass range. MALDI can routinely analyze compounds from a mass of 700 to 200,000 Daltons or greater. The fact that MALDI predominantly generates ions that are singly charged makes it easy to identify individual molecules. MALDI is also the most reliable technique for analyzing glycoproteins, where the extreme broadness in their peaks reflects the carbohydrate heterogeneity in the proteins Fig. 9. 


\section{Peptide and protein analysis by electrospray}

ESI is also useful for peptide and protein analysis for the following reasons:

(1) compatibility with liquid chromatography,

(2) compatibility with tandem mass analysis,

(3) high sensitivity (subpicomole with nanoESI),

(4) ESI allows for multiple charging and therefore the analysis of proteins with limited $\mathrm{m} / z$ range analyzers,

(5) multiple charging allows for more complete fragmentation in tandem MS experiments.

MALDI and ESI are very different types of ionization sources for peptide and protein analysis, with each having specific advantages over the other. Perhaps the most important of these advantages include MALDI's ability to handle complex mixtures while ESI can be directly interfaced with liquid chromatography. In terms of their performance with standard analyzers (TOF for MALDI and quadrupoles/quadrupole ion traps for ESI) they are quite comparable in the low mass range while for protein analysis ESI-quadrupoles tend to provide better resolution and accuracy (Fig. 10).

As with MALDI, the electrospray ionization of peptides and proteins involves the addition of a proton or multiple protons. Sample preparation is achieved by dissolving the sample in a protic volatile solvent system that is relatively homogeneous and contains less than one millimolar concentration of salt although higher concentrations have been used (as high as $100 \mathrm{mM}$ with $\mathrm{NH}_{4} \mathrm{Ac}$ ). However, some salts (alkali and alkaline) and phosphate buffers are more detrimental to signal. Because of the good mass accuracy/stability associated with quadrupole analyzers and because ESI can be easily interfaced with liquid chromatography, electrospray is often the method of choice for peptides and pure low molecular weight proteins below 30,000 Da. Sample spectra are shown in Figs 11-13. There are only a few preparation procedures and no matrices for electrospray, making it a quick method for obtaining molecular weight information although more sample is typically consumed ( $\sim 1$ picomoles) than with MALDI $(<1$ picomole). With respect to ESI, greater sensitivity can be obtained if nanoelectrospray [11] is used.

Another important feature of electrospray is its ability to generate multiply charged species. Multiple charging makes it possible to observe large proteins with mass analyzers that have a relatively small mass range. In addition, observing multiple peaks for the same peptide allows one to make multiple molecular weight calculations from a single spectrum. Thus one can average these values and obtain a more accurate molecular weight. The question often arises, especially concerning the analysis of peptides and proteins,

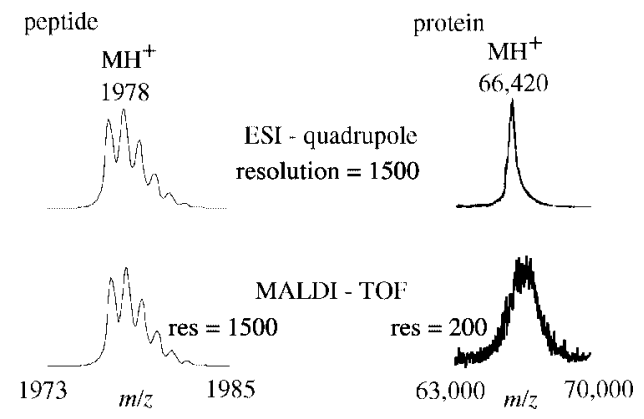

Fig. 10. A direct comparison of peptide and protein data obtained from an ESI-quadrupole and MALDI-TOF mass spectrometers. The data for the peptide is quite comparable while the extra broadness of the peak associated with the protein MALDI-TOF spectrum reflects its lower resolution and accuracy in the higher mass range, when it is compared to the deconvoluted ESI spectrum. 


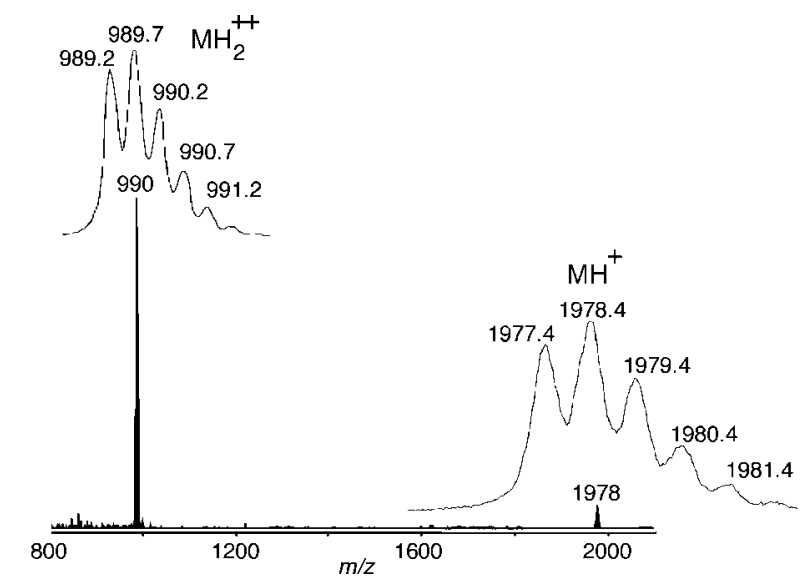

$\begin{array}{cc}\begin{array}{c}\text { (molecular mass + \#protons)/charge }= \\ \text { doubly charged }\end{array} & \text { mass-to-charge ratio } \\ \text { singly charged } \\ (1976.4+2.0) / 2=989.2 & (1976.4+1.0) / 1=1977.4 \\ (1977.4+2.0) / 2=989.7 & (1977.4+1.0) / 1=1978.4 \\ (1978.4+2.0) / 2=990.2 & (1978.4+1.0) / 1=1979.4 \\ (1979.4+2.0) / 2=990.7 & (1979.4+1.0) / 1=1980.4\end{array}$

Fig. 11. The question of whether a peptide (or any other species) observed in the mass spectrum is singly, doubly, or multiply charged can be addressed in a several ways. In this spectrum it is possible to look at the isotopic distribution and determine the spacing between the isotopes. If isotopes are separated by one mass unit the charge is $1+$, if by $1 / 2$ mass units, the charge is $2+$. The calculations further demonstrate that the charge state can be determined from the isotope spacing.
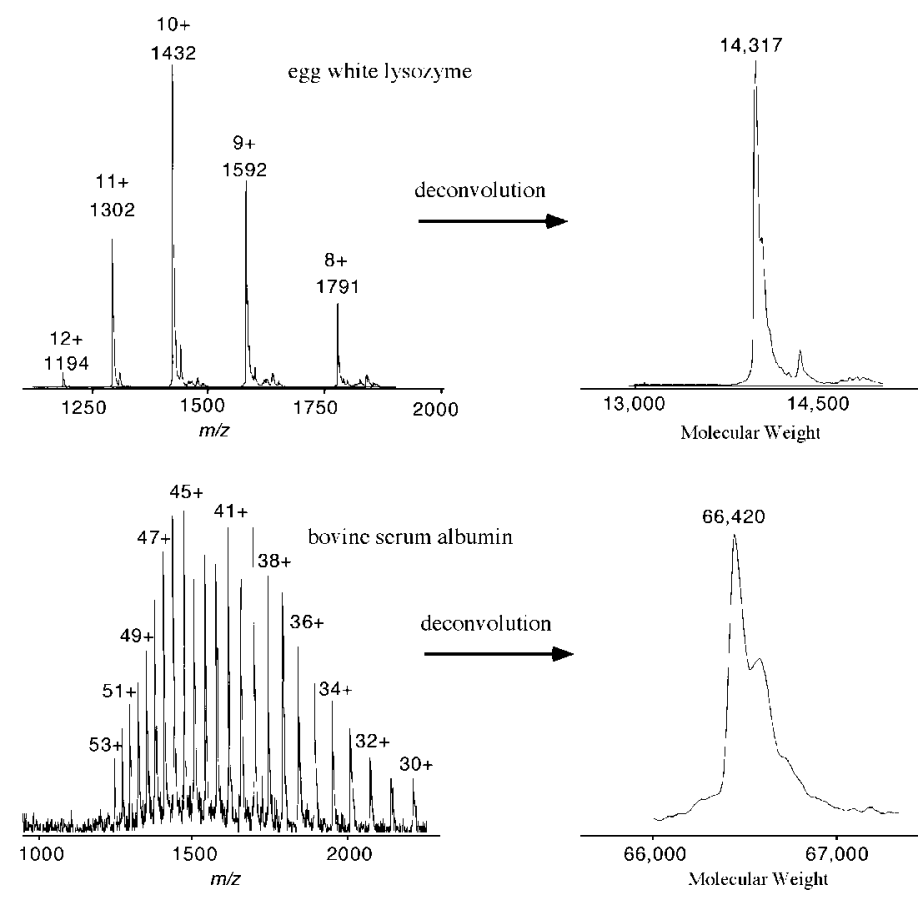

Fig. 12. Electrospray mass spectra with the deconvoluted molecular weight spectrum of egg white lysozyme and bovine serum albumin (BSA). 


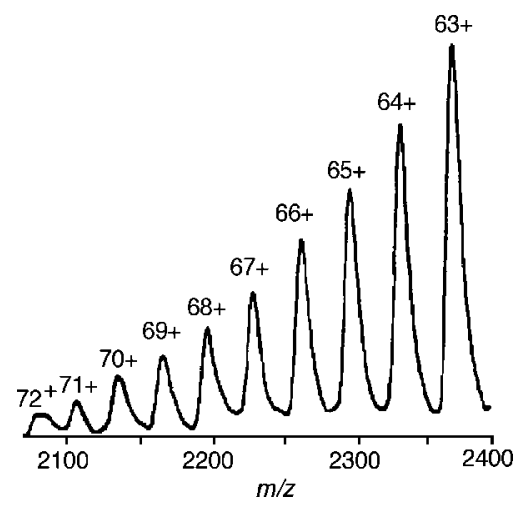

Fig. 13. Electrospray mass spectrum of a large glycoprotein IgG-class murine monoclonal antibody, anti-(human a1-acid glycoprotein). Averaged molecular weight $=149,599 \pm 12 \mathrm{Da}$. Sample consumption was 99 pmol. Adapted from [24].

"How is the charge state of the observed ion determined?", there are two ways: (1) is by monitoring the isotope pattern and (2) is looking at adjacent charge states. In the spectrum in Fig. 11 two peaks are seen, one at $m / z 1978$ and another at $m / z$ 990. How does the investigator know these peaks correspond to the same compound? Since most electrospray spectrometers have good resolution, it is often possible to look at the isotopic distribution of singly and doubly charged ions. By looking at the isotopic distribution of the above ions (Fig. 11) we can readily see the isotopes are separated by one mass unit at $m / z 1978$ and $1 / 2$ mass units at $m / z 990$. Each pattern therefore corresponds to the $1+$ and $2+$ charge states, respectively. This is due to the fact that the mass spectrometer measures the mass-to-charge ratio. Therefore, as the calculation demonstrates, for the $2+$ charge state the isotopes are separated by $1 / 2$, at $3+$ the isotopes are separated by $1 / 3$, and at $4+$ the isotopes are separated by $1 / 4$. Unfortunately, the resolving power of the common quadrupole electrospray mass spectrometers only allow for distinguishing between singly and doubly charged species. While in some cases triply and quadruply charged species can be identified.

Fortunately, as bigger peptides and proteins are analyzed, a distribution of ions is obtained (Fig. 12). Even though we cannot look at the individual charge states from the isotopic pattern, since the resolving power of many instruments will not allow this, we can deduce the charge state by looking at a couple of peaks in the distribution. However, it is usually unnecessary to perform these calculations since all commercial instruments allow for these computations to be performed very easily. Figure 12 illustrates the results of two computer-generated calculations.

Another advantage of generating multiply charged ions with electrospray is that multiply charged peptide ions tend to give more complete fragmentation spectra. This has become increasingly important for de novo sequencing of peptides [12,13].

One of the limitations of ESI in comparison to MALDI is its lower sensitivity; however this has been overcome with the commercial introduction of nanoelectrospray ionization sources. NanoESI is a slight variation on ESI such that the spray needle has been made very small and positioned very close to the entrance of the vacuum of the mass spectrometer and the mass analyzer. The end result is increased efficiency and thus greatly reduced sample amounts are needed. For instance, the flow rates for nanoESI sources are on the order of tens of nanoliters per minute. Another advantage of this technique is that the droplets are typically formed smaller than normal ESI and thus nanoESI is more tolerant of salts and other impurities. This approach has become particularly important in protein and peptide analysis where only small quantities of material are available. 
HPLC

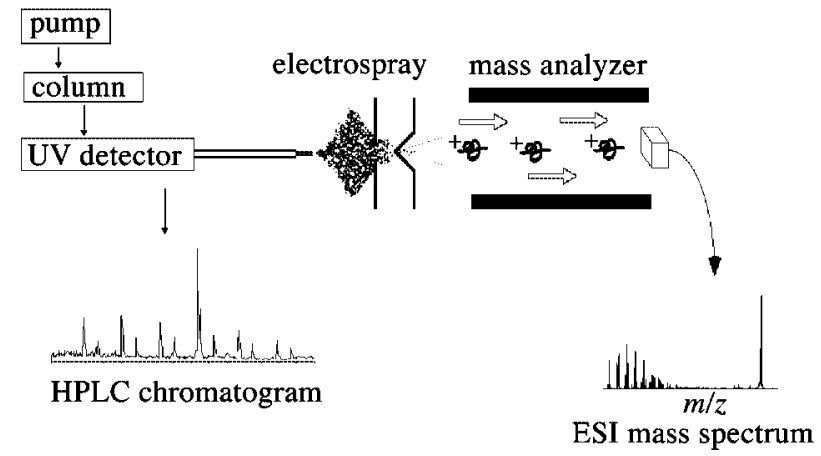

Fig. 14. Interfacing liquid chromatography with electrospray ionization mass spectrometry.

ESI can also be applied to glycoprotein analysis, in fact one of the largest proteins observed with ESIMS has been with a glycoprotein. However, direct analysis of glycoproteins by electrospray provides only limited information, and requires very high purity because excessively heterogeneous compounds produce complicated spectra, often making interpretation difficult or impossible. In addition, sample heterogeneity will often reduce instrument sensitivity. In general electrospray is not the method of choice and MALDI is typically used for the analysis of glycoproteins (Fig. 9).

Another important feature of ESI-MS is its ability to directly analyze compounds from aqueous or aqueous/organic solutions, a feature that has established the technique as a convenient mass detector for liquid chromatography (LC) Fig. 14. ESI with quadrupole or ion trap analyzers [14-16] also allows for MS analysis at relatively high LC flow rates $(1.0 \mathrm{ml} / \mathrm{min})$ and high mass accuracy $( \pm 0.01 \%)$, adding a new dimension to the capabilities of LC characterization. In fact, using ESI-MS as a detector for LC was one of its first obvious applications. Numerous reports have been published on this with special emphasis on small molecule, peptide, and protein analysis [17-20]. The combination of LC and ESI-MS is excellent for routine and reproducible molecular weight determinations on a wide variety of compounds, whether they are positively (i.e., peptides and proteins) or negatively (i.e., oligonucleotides) charged. LC/MS and especially LC/MS/MS has found significant utility in protein identification and peptide de novo sequencing [21].

ESI induces ion formation from small droplets, once the ions are formed they are subject to collisions before entering the mass analyzer. These collisions can decluster aggregates, induce fragmentation, and change the charge states by removing protons. The energy with which the ions enter the mass analyzer through the orifice can determine the amount of fragmentation that will take place. This energy can be adjusted by varying the electrospray declustering or fragmentation potential. A diagram of the orifice is shown in Fig. 15. However it should be pointed out that this design is variable depending on the instrument yet the same effect is observed in virtually all electrospray mass spectrometers. The energy of the ions entering the orifice is determined by the voltage applied to the orifice and the higher the energy, the more fragmentation is observed. In addition to inducing peptide fragmentation increasing the declustering potential can cause the charge distribution of a protein to change. This is related to the protein's greater collisional energy when entering the mass spectrometer. At higher declustering potentials, more protons are stripped from the protein, causing a shift in the charge state distribution. Figure 15 demonstrates that at a high declustering potential, protons are stripped from egg white lysozyme to produce a charge distribution completely different from one observed at a lower potential. 

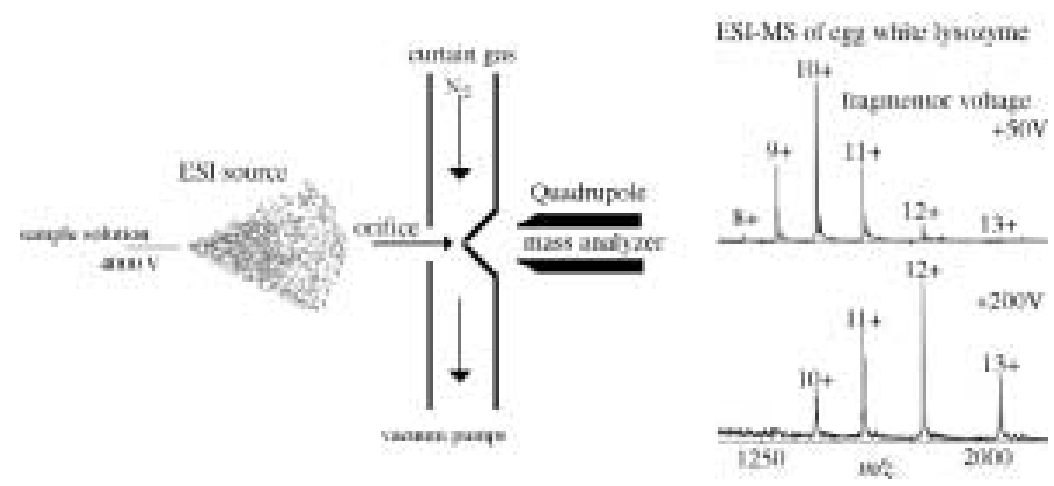

Fig. 15. Orifice-induced collisions are controlled by the potential between the orifice and the quadrupole mass analyzer. The variation of this potential can affect sensitivity, ability to observe noncovalent interactions, fragmenation, and charge state. The potential difference between the orifice and the quadrupole affects the energy of collisions prior to mass analysis. Electrospray mass spectra of egg white lysozyme at two different orifice (declustering) potentials demonstrating that at higher potentials protons can be stripped from the protein thereby shifting the charge state distribution.

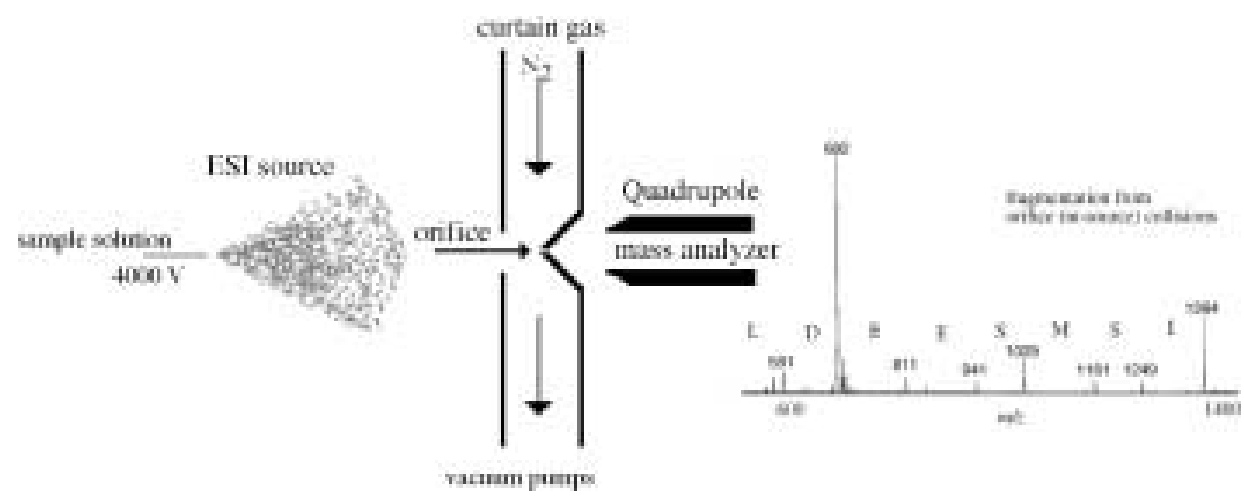

Fig. 16. Collisions at the orifice can be useful for obtaining structural information. The collisions of this peptide, $\mathrm{H}$-[ISMSEEDLLNAK]-OH, have resulted in the formation of fragment ions from the N-terminal directly corresponding to its sequence. It is important to note that this is not a tandem mass spectrometry experiment and the fragment ions were generated as the ions entered the mass analyzer.

Another interesting aspect of the ESI source is that it can induce fragmentation of small peptides typically below a mass of $3000 \mathrm{Da}$. In relation to peptide/protein analysis the declustering potential allows for a small amount of fragmentation, thus allowing for sequencing information to be obtained. Figure 16 illustrates how useful sequence information was obtained on a peptide simply by increasing this potential. A drawback of the application is that it can only be used on very pure samples; if the samples are not pure it may be easy to mistake an impurity for a fragment ion. When using orificeinduced fragmentation, identifying true fragment ions versus impurities is difficult. This problem can be overcome with the use of a tandem mass spectrometer [22,23]. Sample purity is not as significant an issue with a tandem mass spectrometer since an ion of interest can be isolated even in the presence of several other ions (peptides). Once the ion of interest is isolated, it can be exposed to collisions and the resultant fragment ions can be mass analyzed. This technique is called tandem mass analysis. 


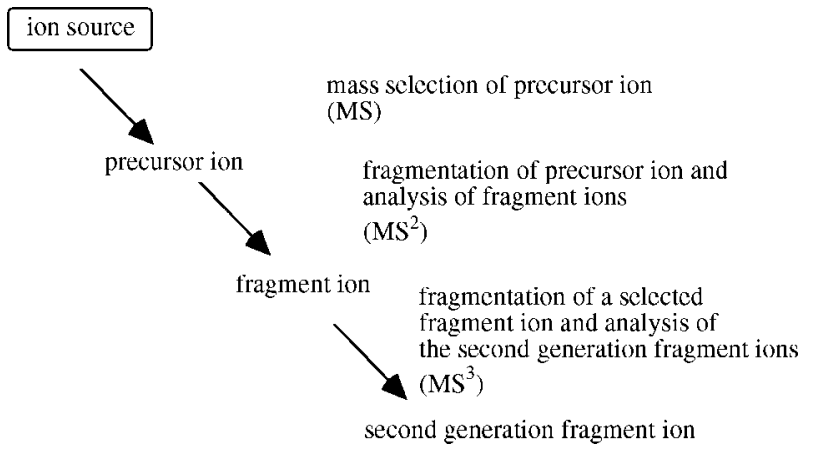

Scheme 1. Description of tandem mass spectrometry experiments.

\section{Overview}

The analysis of peptides and proteins by mass spectrometry techniques such as ESI and MALDI has changed the way biochemists are approaching the challenges of characterizing protein expression in the post-genomic era. The availability of these sensitive and gentle ionization methods has made it possible to analyze even labile post-translationally modified (PTM) proteins and peptides through a range of available mass analyzers including TOFs, triple quadrupole, quadrupole ion traps, FTMS, and hybrid instruments. Tandem mass spectrometry experiments are allowing researchers to ascertain structural information on proteins and peptides, and to identify post-translational modifications. Mass spectrometry is now routinely used in labs around the world as a fundamental tool for identifying unknown proteins through MS and tandem MS analysis of proteolysis products, followed by computer-aided genomic database searching. The high resolution and mass accuracy afforded by modern mass spectrometers have greatly facilitated its use for protein identification. Recent developments in front-end techniques such as $2 \mathrm{D}$ nano-LC electrospray mass spectrometry and isotope-coded affinity tag (ICAT) are now allowing researchers to use mass spectrometry to characterize and quantify changes in protein expression of highly complex cellular systems. Understanding the etiology of disease states through protein expression profiling is the first step towards the development of new and more effective drugs for treating these conditions.

\section{References}

[1] R.B. Cole, ed., Electrospray Ionization Mass Spectrometry: Fundamentals, Instrumentation, and Applications, John Wiley and Sons, New York, 1997.

[2] J.B. Fenn, M. Mann, C.K. Meng, S.F. Wong and C.M. Whitehouse, Electrospray ionization for mass spectrometry of large biomolecules, Science 246 (1989), 64-71.

[3] G.A. Valaskovic, N.L. Kelleher, D.P. Little, D.J. Aaserude and F.W. McLafferty, Attomole-sensitivity electrospray source for large-molecule mass spectrometry, Analyt. Chem. 67 (1995), 3802-3805.

[4] M. Karas and F. Hillenkamp, Laser desorption ionization of proteins with molecular weight exceeding 10,000 daltons, Analyt. Chem. 60 (1988), 2299-3201.

[5] O.N. Jensen, A. Podtelejnikov and M. Mann, Delayed extraction improves specificity in database searches by matrixassisted laser desorption/ionization peptide maps, Rapid Comm. Mass Spectrom. 10 (1996), 889-896.

[6] J. Wei, J.M. Buriak and G. Siuzdak, Desorption-ionization mass spectrometry on porous silicon, Nature 399 (1999), 243-246.

[7] S.D. Patterson and R. Aebersold, Mass spectrometric approaches for the identification of gel-separated proteins, Electrophoresis 16 (1995), 1791-1814. 
[8] M. Kinter and N.E. Sherman, Protein Sequencing and Identification Using Tandem Mass Spectrometry, John Wiley and Sons, New York, 2000.

[9] G. Neubauer and M. Mann, Mapping of phosphorylation sites of gel-isolated proteins by nanoelectrospray tandem mass spectrometry: Potentials and limitations, Analyt. Chem. 71 (1999), 235-242.

[10] T.L. Quenzer, M.R. Emmet, C.L. Hendrickson, P.H. Kelly and A.G. Marshall, High sensitivity Fourier transform ion cyclotron resonance mass spectrometry for biological analysis with nano-LC and microelectrospray ionization, Analyt. Chem. 73 (2001), 1721.

[11] M. Wilm, A. Shevchenko, T. Houthaeve, S. Breit, L. Schweigerer, T. Fotsis and M. Mann, Femtomole sequencing of proteins from polyacrylamide gels by nano-electrospray mass spectrometry, Nature 379 (1996), 446-469.

[12] X.-J. Tang and R.K. Boyd, An investigation of fragmentation mechanisms of doubly charged protonated tryptic peptides, Rapid Comm. Mass Spectrom. 6 (1991), 651-657.

[13] T.R. Covey, E.C. Huang and J.D. Henion, Structural characterization of protein tryptic peptides via liquid chromatography/mass spectrometry and collision-induced dissociation of their doubly charged ions, Analyt. Chem. (1991), 193-200.

[14] R.W. Vachet, K.L. Ray and G.L. Glish, Origin of product ions in the MS/MS spectra of peptides in a quadrupole ion trap, J. Am. Soc. Mass Spectrom. 8 (1998), 341-344.

[15] R.E. March and J.F.J. Todd, eds, Practical Aspects of Ion Trap Mass Spectrometry, Vol. I. Fundamentals of Ion Trap Mass Spectrometry, CRC Press, Boca Roaton, 1995

[16] K.R. Jonscher and J.R. Yates, III, The quadrupole ion trap mass spectrometer - A small solution to a big challenge, Analyt. Biochem. 244 (1997), 1-15.

[17] M.E. Davis, D.C. Stahl, S.A. Hefta and T.D. Lee, A microscale electrospray interface for on-line, capillary liquid chromatography/tandem mass spectrometry of complex peptide mixtures, Analyt. Chem. 67 (1995), 4549-4556.

[18] M.T. Davis and T.D. Lee, Rapid protein identification using a microscale electrospray LC/MS system on an ion trap mass spectrometer, J. Am. Soc. Mass Spectrom. 9 (1998), 194-201.

[19] R.A. Yost and C.G. Enke, Selected ion fragmentation with a tandem quadrupole mass spectrometer, J. Am. Chem. Soc. 100 (1978), 2274-2275.

[20] W.E. Haskins, Z. Wang, C.J. Watson, R.R. Rostand, S.R. Witowski, D.H. Powell and R.T. Kennedy, Capillary LC-MS2 at the attomole level for monitoring and discovering endogenous peptides in microdialysis samples collected in vivo, Analyt. Chem. 73 (2001), 5005-5015.

[21] A. Shevchenko, I. Chernushevich, W. Ens, K.G. Standing, B. Thomson, M. Wilm and M. Mann, Rapid de novo peptide sequencing by a combination of nanoelectrospray, isotopic labeling, and a quadrupole/time-of-flight mass spectrometer, Rapid Comm. Mass Spectrom. 11 (1997), 1015-1024.

[22] F.W. McLafferty, Tandem mass spectrometry, Science 214 (1981), 280-287.

[23] I.A. Papayannopoulos, The interpretation of collision-induced dissociation tandem mass spectra of peptides, Mass Spectrom. Rev. 14 (1995), 49-73.

[24] R. Feng and Y. Konishi, Analysis of antibodies and other large glycoproteins in the mass range of 150000-200000 Da by electrospray ionization mass spectrometry, Analyt. Chem. 64 (1992), 2090-2095. 


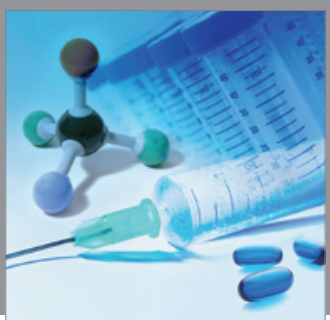

International Journal of

Medicinal Chemistry

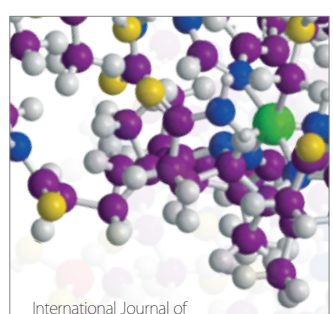

Carbohydrate Chemistry

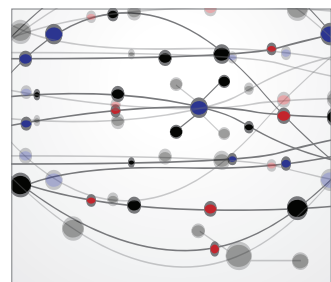

The Scientific World Journal
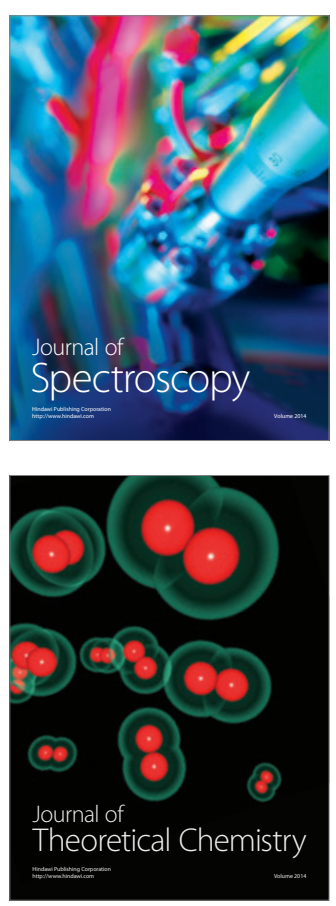
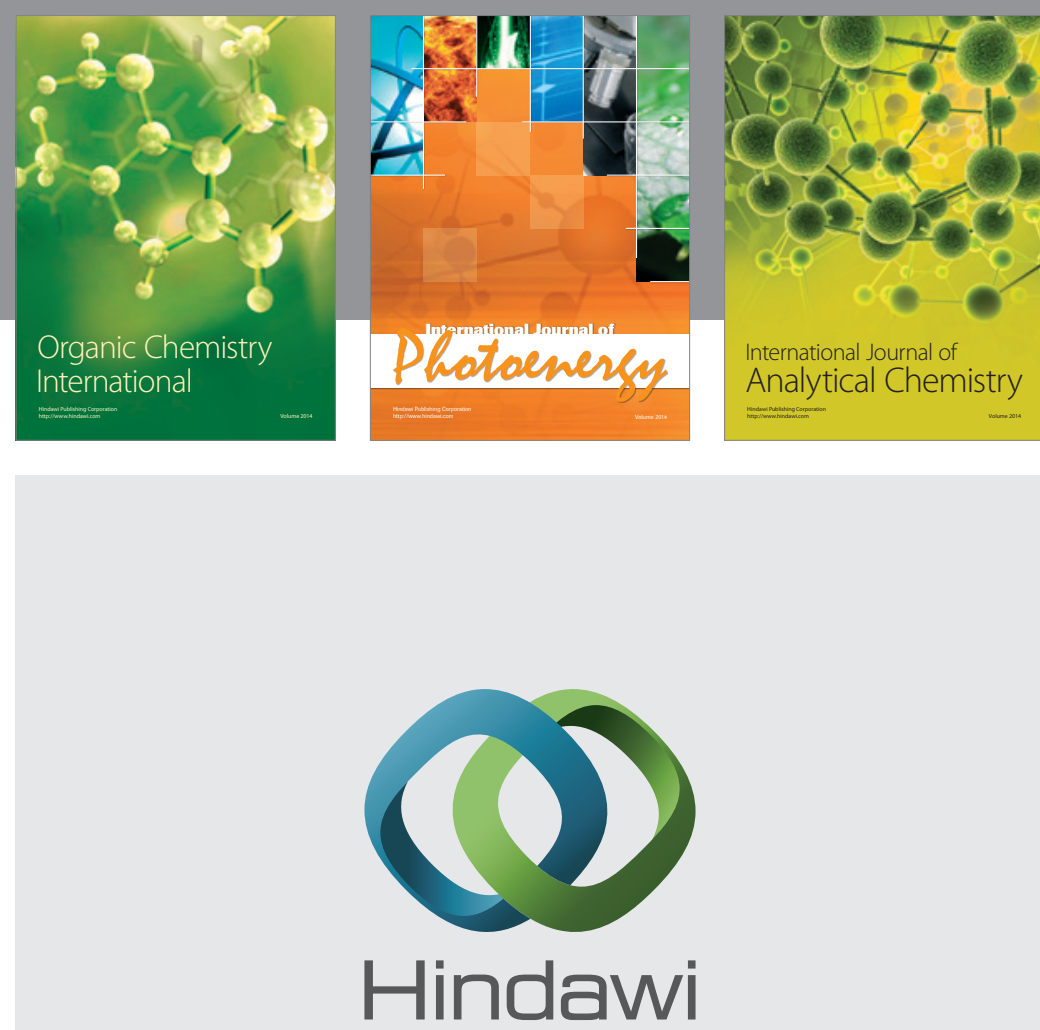

Submit your manuscripts at

http://www.hindawi.com
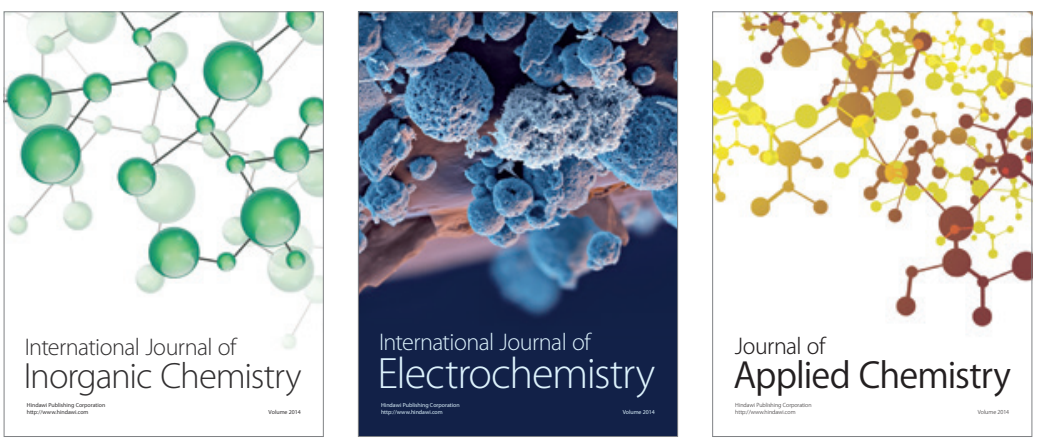

Journal of

Applied Chemistry
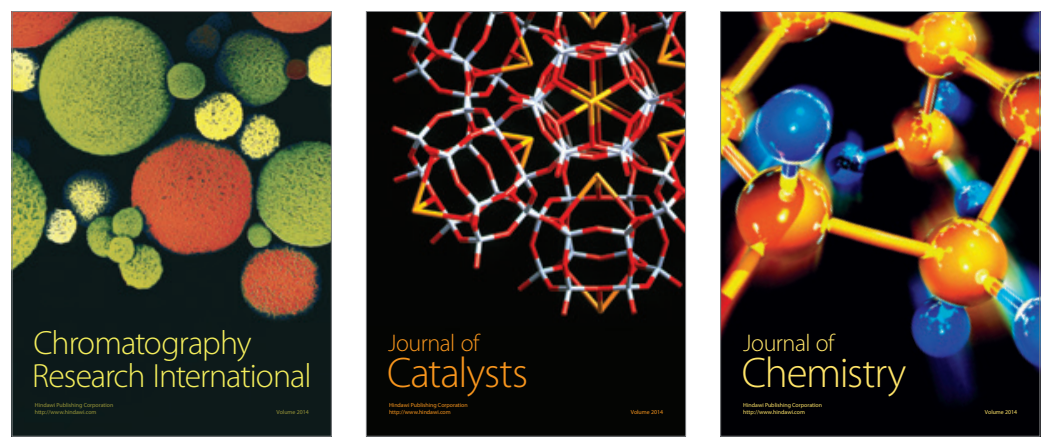
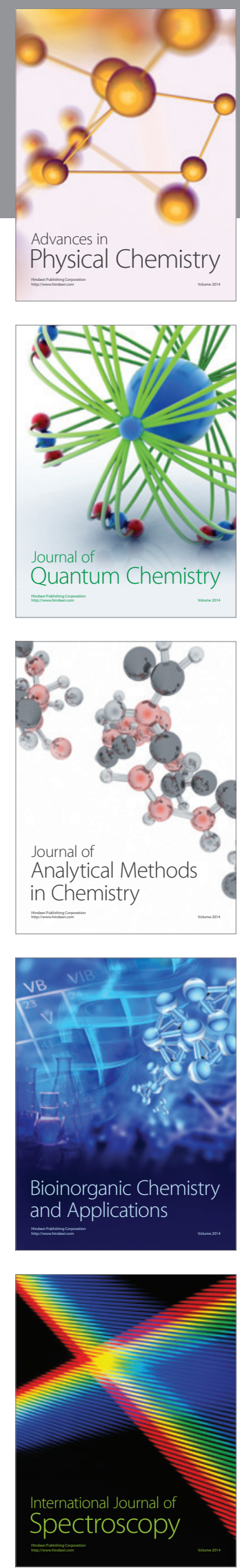